\title{
Pulmonary blastomycosis in a professional diver: An occupational risk
}

\author{
Ryan R Kroll MD(c) ${ }^{1}$, Ronald F Grossman MD FRCPC 2,3
} RR Kroll, RF Grossman. Pulmonary blastomycosis in a professional
diver: An occupational risk. Can Respir J 2013;20(5):340-342.

In certain parts of the United States and Canada, and northern Ontario in particular, the dimorphic fungus Blastomyces dermatitidis is endemic and can cause infection in exposed individuals. The site of infection is usually pulmonary, causing respiratory and constitutional symptoms, but can also affect other sites in the body. Symptom severity can vary substantially from no symptoms to fatal acute respiratory distress syndrome. The present report describes a 27-year-old professional diver who had recently worked in northern Ontario, who developed symptoms of pneumonia and exhibited atypical findings on chest imaging. He was diagnosed with blastomycosis based on histopathological findings and fungal culture, and was treated with amphotericin B and itraconazole in accordance with treatment guidelines. While outdoor occupations in endemic areas increase the risk of infection, there is no literature specifically identifying professional diving as an occupational risk for blastomycosis.

Key Words: Atypical pneumonia; Blastomycosis; Occupational exposure

Learning objectives:

- To be aware that Blastomyces dermatitidis is found throughout Ontario, with higher frequency recorded in northern regions.

- To recognize that a detailed social/occupational history is crucial in informing diagnostic investigations and treatment in patients presenting with pneumonia-like symptoms.

Can MEDS competency: Medical Expert

Pretest:

- In what environmental conditions does B dermatitidis thrive?

- What is the primary method of diagnosing pulmonary blastomycosis?

\section{CASE PRESENTATION}

A 27-year-old man presented to an Ontario hospital with a two-week history of pleuritic left upper chest pain, night sweats and a $4.5 \mathrm{~kg}$ weight loss over the span of one month. Two days before the visit, he developed hemoptysis. He had been diagnosed with pneumonia four times in the preceding 2.5 years. He was a former smoker who had childhood asthma. His medical history was otherwise unremarkable. He had been working in northern Ontario, performing underwater construction as a professional diver. In this occupation, he had significant exposure to rotting wooden docks, wet soil, raw sewage, old buildings, bats and other animals. Family history was notable for a malignancy in his father that metastasized to the lungs. His temperature was $36.7^{\circ} \mathrm{C}$ (oral), heart rate 83 beats/min, blood pressure 141/85 mmHg and oxygen saturation $98 \%$ on room air. Physical examination revealed faint crackles in the left upper lobe. He was admitted to the department of internal medicine and was started on ceftriaxone and azithromycin.

Bloodwork on admission demonstrated a white blood cell count of $15.7 \times 10^{9} / \mathrm{L}$, with a neutrophilia of $11.5 \times 10^{9} / \mathrm{L}$. Sputum cytology was negative for malignant cells, and bronchial cytology was negative for malignancy and granulomas. Initially, there was no fungal growth in

\section{Une blastomycose pulmonaire chez un plongeur professionnel : un risque lié au travail}

Dans certaines parties des États-Unis et du Canada, notamment au nord de l'Ontario, le champignon dimorphe Blastomyces dermatitidis est endémique et peut provoquer une infection chez les personnes qui y sont exposées. L'infection a généralement un foyer pulmonaire, provoquant des symptômes respiratoires et constitutionnels, mais peut également toucher d'autres foyers de l'organisme. La gravité des symptômes peut varier considérablement, passant de l'absence de symptômes à un syndrome de détresse respiratoire aiguë sévère. Le présent rapport décrit le cas d'un plongeur professionnel de 27 ans qui avait récemment travaillé dans le nord de l'Ontario, a contracté des symptômes de pneumonie et présenté des manifestations atypiques à l'imagerie pulmonaire. On lui a diagnostiqué une blastomycose selon les observations histopathologiques et la culture fongique, laquelle a été traitée à l'amphotéricine $\mathrm{B}$ et à l'itraconazole conformément aux directives thérapeutiques. Bien qu'un travail extérieur dans les régions endémiques accroisse le risque d'infection, aucune publication n'indique expressément le plongeon professionnel comme risque professionnel de blastomycose.

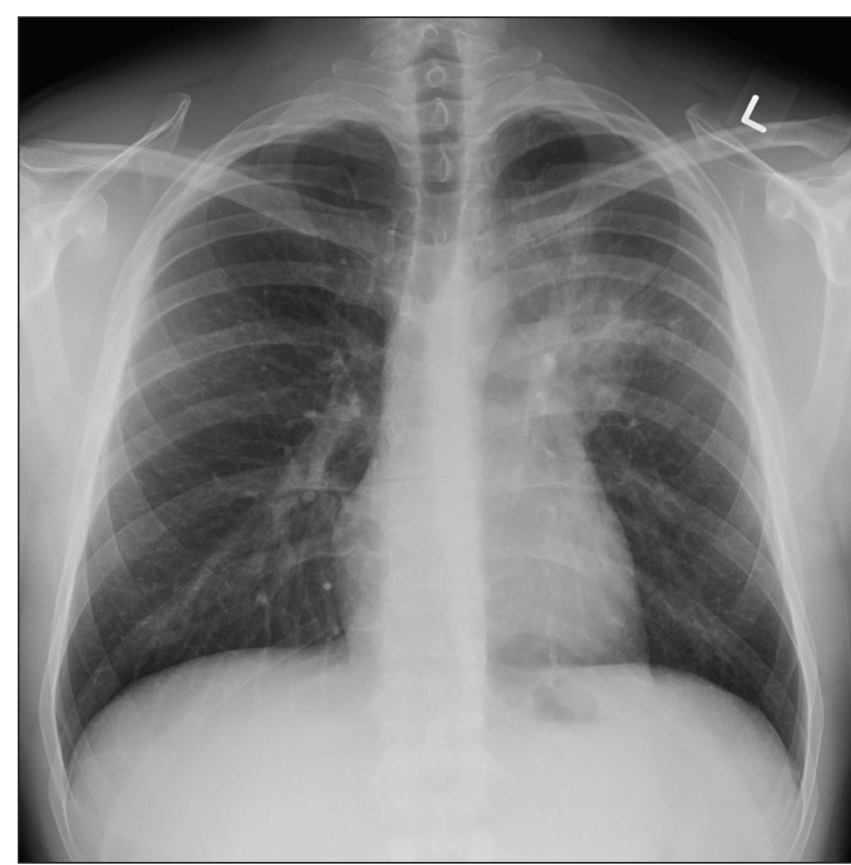

Figure 1) Posterior-anterior view chest radiograph showing consolidation and volume loss in the left upper lobe

the bronchial wash, but the laboratory eventually isolated budding yeast cells weeks later. Polymerase chain reaction was negative for tuberculosis. A transbronchial biopsy was performed, demonstrating noncaseating granulomas and Schaumann bodies.

A chest $\mathrm{x}$-ray demonstrated consolidation and volume loss in the left upper lobe compatible with pneumonia (Figure 1). A computed tomography scan of the chest showed a dense, left upper lobe mass measuring $7.3 \mathrm{~cm} \times 4.8 \mathrm{~cm}$ with truncated bronchi extending into it along its 


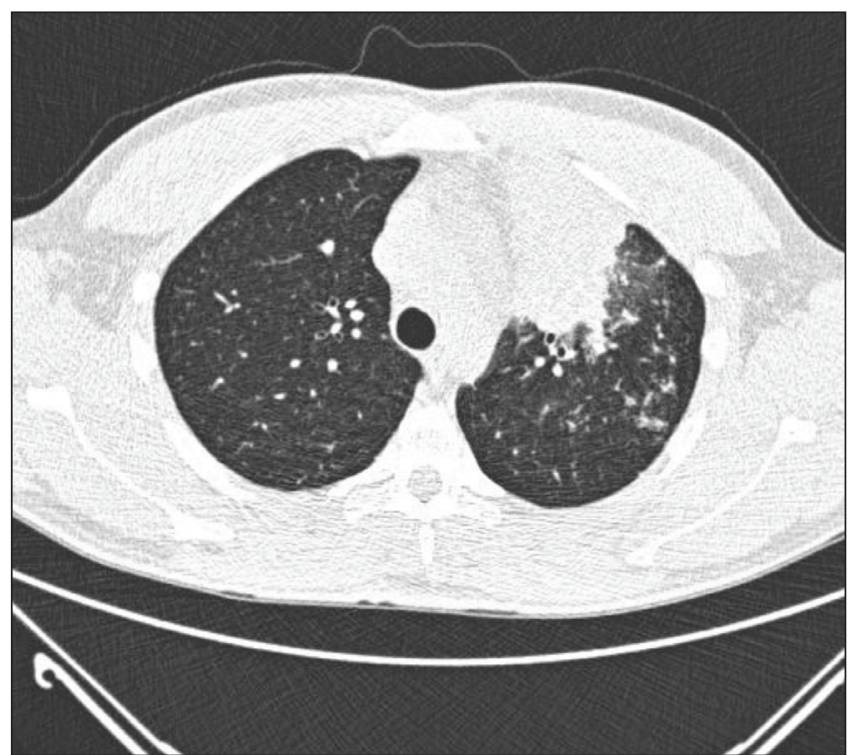

Figure 2) Computed tomography scan showing a dense, left upper lobe mass measuring $7.3 \mathrm{~cm} \times 4.8 \mathrm{~cm}$ with truncated bronchi extending into it along its periphery; the mass is contiguous with surrounding structures. No significant adenopathy is apparent

periphery; the mass was contiguous with surrounding structures (Figure 2). The computed tomography scan also revealed small foci of nodular density in the left upper lobe with patchy bronchovascular markings and no significant adenopathy. Initially concerned about possible malignancy, the radiologist's final diagnosis was that of atypical pneumonia.

Wedge biopsies demonstrated necrotizing granulomatous inflammation with fibrovascular proliferation and fibrosis (Figure 3). The necrotic centres were noncaseating with neutrophillic infiltrates, and granulomas were composed primarily of macrophages accompanied by smaller numbers of lymphocytes and giant cells. These findings were consistent with blastomycosis infection. Methenamine silver staining as well as periodic acid-Schiff staining of the samples both showed rounded thick-walled structures $11 \mu \mathrm{m}$ to $15 \mu \mathrm{m}$ in diameter, consistent with blastomycosis (Figure 4). B dermatitidis was eventually confirmed by culture.

The patient was diagnosed with blastomycosis and treated with amphotericin $\mathrm{B}(\mathrm{AmB})$ followed by itraconazole. He was released from hospital and followed as an outpatient by specialists from the departments of infectious diseases and respirology.

\section{DISCUSSION}

Blastomycosis is a granulomatous disease caused by inhalation of conidia from the dimorphic fungus $B$ dermatitidis, and was first documented in Canada in 1910 (1,2). B dermatitidis is endemic to North America, particularly the midwestern and southern United States, as well as areas of Canada that border bodies of water such as the Great Lakes and the St Lawrence Seaway (1). Northern Ontario has a particularly high incidence of blastomycosis compared with the rest of Ontario, with $61 \%$ of the province's diagnosed cases of blastomycosis between 1994 and 2003 (2). The incidence of 117.2 cases per 100,000 population in northern Ontario is the highest in North America (2).

Most commonly, B dermatitidis infection occurs in individuals who are exposed to soil contaminated with the fungus. It is often found in soil rich in decaying vegetation, animal droppings and rotting wood $(1,3)$. Individuals who live in densely wooded areas with moist soil, close to bodies of fresh water are at an increased risk of developing blastomycosis, as are those with occupations that involve spending time in these outdoor environments. These risk factors are well documented in the literature (3); however, a PubMed search using keywords

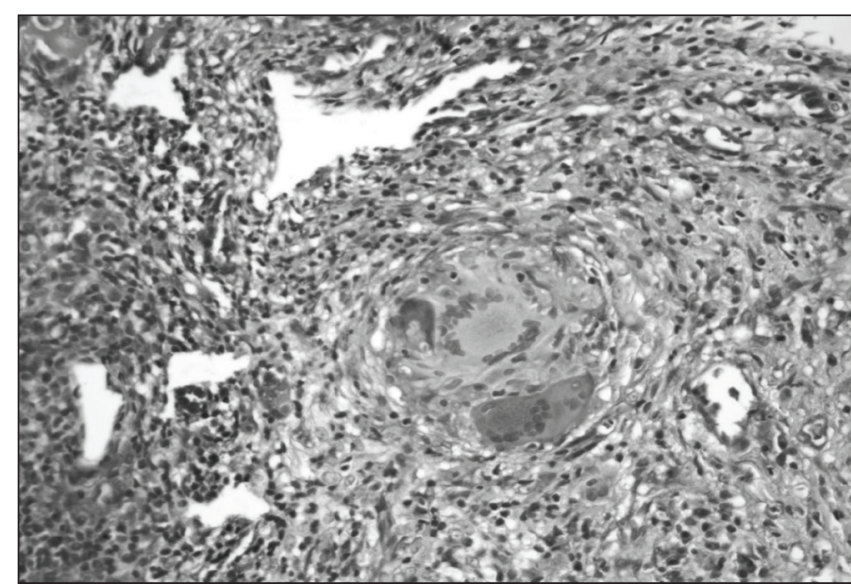

Figure 3) Necrotizing, noncaseating granulomatous inflammation with fibrovascular proliferation and fibrosis (periodic acid-Schiff stain, original magnification $\times 320$ )

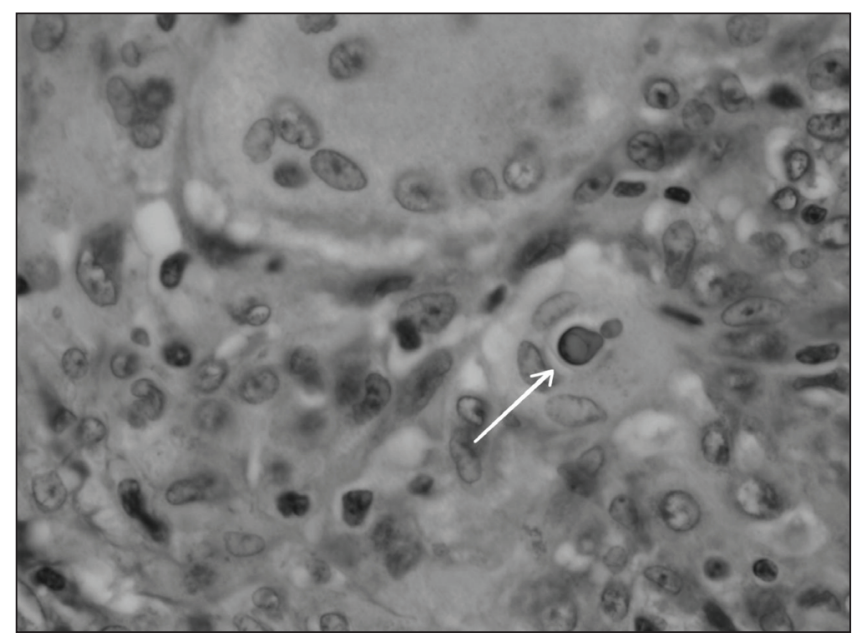

Figure 4) Rounded, thick-walled structures $11 \mu \mathrm{m}$ to $15 \mu \mathrm{m}$ in diameter (arrow) on periodic acid-Schiff staining, consistent with blastomycosis (original magnification $\times 640$ )

"blastomycosis and occupation" did not reveal research that identified professional diving as an occupational risk factor for the disease.

Pulmonary infection is the most common manifestation of blastomycosis and is found primarily in males. It presents with cough, sputum, hemoptysis, dyspnea, fever, malaise, myalgias, weight loss and pleuritic chest pain $(2,4)$. The severity of infection can range from patients who are asymptomatic (up to 50\%), to those with lifethreatening acute respiratory distress syndrome $(1,3)$. While the most frequent site of infection is the lungs, blastomycosis can manifest as infection of extrapulmonary sites including bones, skin, the genitourinary system and the central nervous system $(2,4)$.

Most patients who present with symptoms of pulmonary blastomycosis demonstrate varied and nonspecific findings on chest $\mathrm{x}$-rays, which can mimic those of bacterial pneumonia, malignancy, acute respiratory distress syndrome or tuberculosis (2). Diagnosis is primarily made on histological examination of sputum or bronchial washings specially treated with a variety of stains including potassium hydroxide, periodic acid-Schiff and methenamine silver stain. Similar staining techniques are used in extrapulmonary disease (1). The typical findings of $B$ dermatitidis are single, broad-based budding yeasts that are $8 \mu \mathrm{m}$ to $15 \mu \mathrm{m}$ in diameter and can be multinucleated (5). Findings are differentiated from cryptococcus and paracoccidioides based on size and capsule-staining characteristics (5). Cultures of respiratory secretions should be grown on a general-purpose growth medium (eg, Sabouraud dextrose agar), as well as other media depending on the 
circumstances (3). Culture, while providing a more definitive diagnosis, takes much longer than histological studies. B dermatitidis, as a cultured mold, has septate hyphae with diameters of $1 \mu \mathrm{m}$ to $2 \mu \mathrm{m}$, and oval or pyriform single-celled conidia $2 \mu \mathrm{m}$ to $4 \mu \mathrm{m}$ in diameter found at the ends of conidiophores (3). While certain features on culture are specific for $B$ dermatitidis, confirmatory testing is still required and can be accomplished using a variety of modalities (3).

Treatment of blastomycosis is dependent on severity, site of involvement and host factors. In mild to moderate pulmonary and disseminated disease, the first-line treatment is itraconazole for six to 12 months in adults (1). For adults with moderate to severe pulmonary and disseminated disease, as well as in the immunosuppressed, treatment is lipid AmB followed by itraconazole (1). For adults with central nervous system infection, the treatment regimen is with higher dosages of AmB with longer-term use of both $\mathrm{AmB}$ and oral azoles (1). Azoles are contraindicated in pregnancy, with treatment consisting solely of lipid $\mathrm{AmB}(1,3)$. Treatment of children with blastomycosis is similar to that of adults, but with lower antifungal doses (1).

The prognosis for those treated for mild to moderate disease is favourable, with a $95 \%$ success rate (1). Elderly patients, and those who are immunocompromised or have extensive disease, tend to experience worse outcomes (1).

The patient discussed in the present report would have had extensive contact with contaminated soil as a diver in northern Ontario, an area endemic for $B$ dermatitidis. Although possibly protected while wearing his diving apparatus, he would have likely been exposed to the fungus when working without his equipment. His occupation was not limited to underwater tasks: he worked along shorelines and was frequently exposed to rotting wood, sewage, animals and old buildings. The occupational history of exposure in an endemic area for $B$ dermatitidis was the key historical finding in making the diagnosis in a patient with symptoms of pneumonia.
Post-test:

- In what environmental conditions does B dermatitidis thrive? $B$ dermatitidis thrives in moist soil, near fresh water bodies, which contains animal droppings, decaying vegetation and rotting wood.

- What is the primary method of diagnosing pulmonary blastomycosis?

Histopathological examination of sputum and bronchial wash is the primary method of diagnosis. Typical findings are single, broadbased budding yeasts $8 \mu \mathrm{m}$ to $15 \mu \mathrm{m}$ in diameter, which may be multinucleated.

ACKNOWLEDGEMENTS: Dr Grossman was an integral member of the in-hospital management team for the patient discussed in the case, and provided all data, hospital records, consult notes, laboratory reports and images used in this report. Dr Grossman also reviewed and revised the manuscript. Ryan Kroll analyzed the hospital records and compiled the manuscript. Both authors approved the final manuscript.

\section{REFERENCES}

1. Chapman SW, Dismukes WE, Proia LA, et al. Clinical practice guidelines for the management of blastomycosis: 2008 update by the Infectious Diseases Society of America. Clin Infect Dis 2008;46:1801-12.

2. Morris SK, Brophy J, Richardson SE, et al. Blastomycosis in Ontario, 1994-2003. Emerg Infect Dis 2006;12:274-9.

3. Saccente M, Woods GL. Clinical and laboratory update on blastomycosis. Clin Microbiol Rev 2010;23:367-81.

4. Crampton TL, Light RB, Berg GM, et al. Epidemiology and clinical spectrum of Blastomycosis diagnosed at Manitoba Hospitals. Clin Infect Dis 2002;34:310-6.

5. Taxy JB. Blastomycosis: Contributions of morphology to diagnosis. Am J Surg Pathol 2007;31:615-23. 


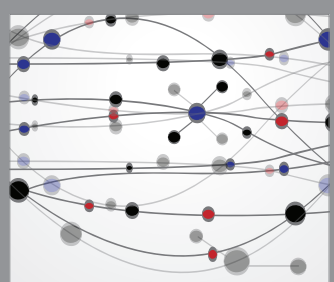

The Scientific World Journal
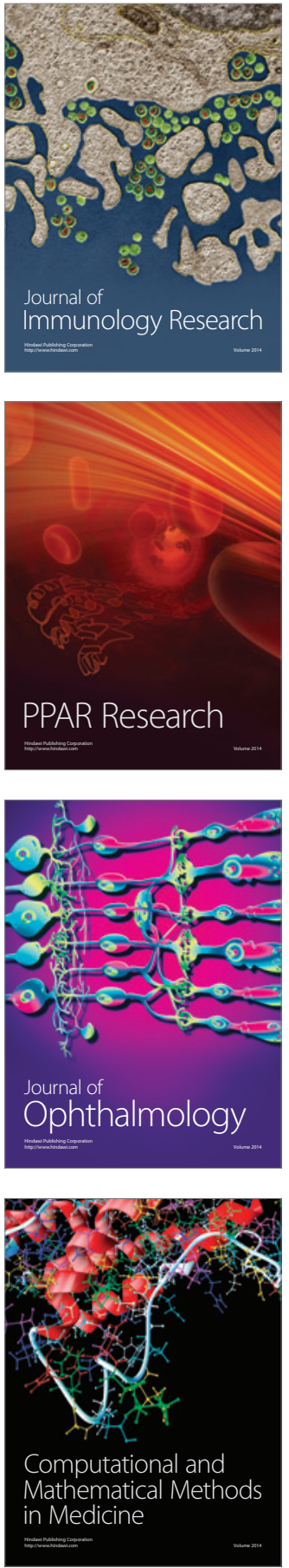

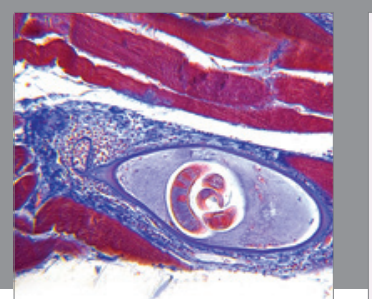

Gastroenterology Research and Practice

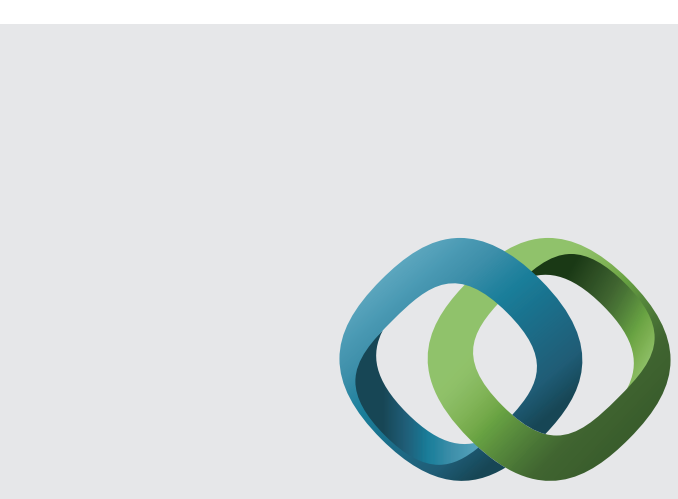

\section{Hindawi}

Submit your manuscripts at

http://www.hindawi.com
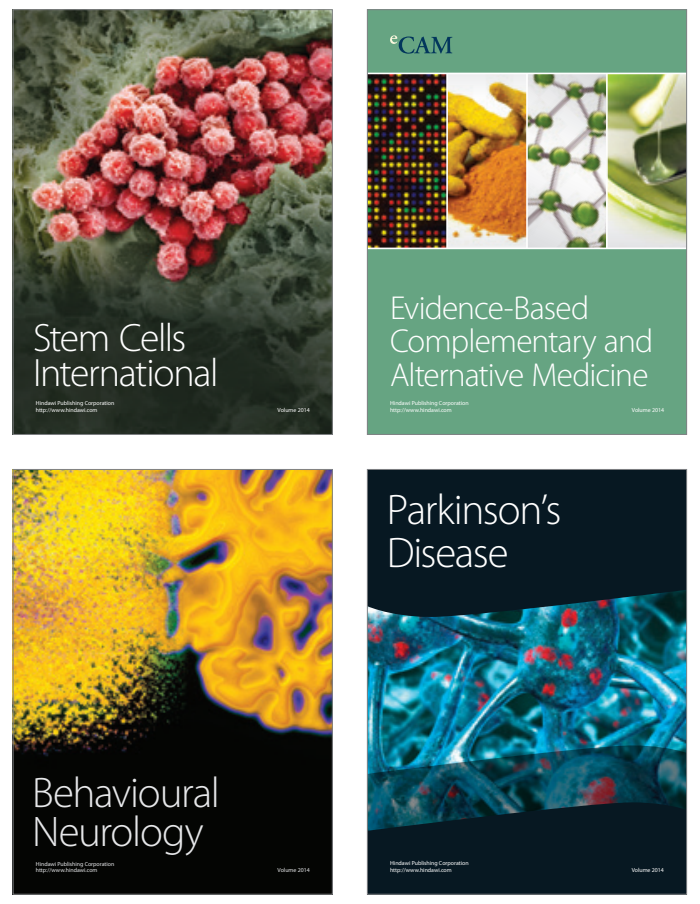
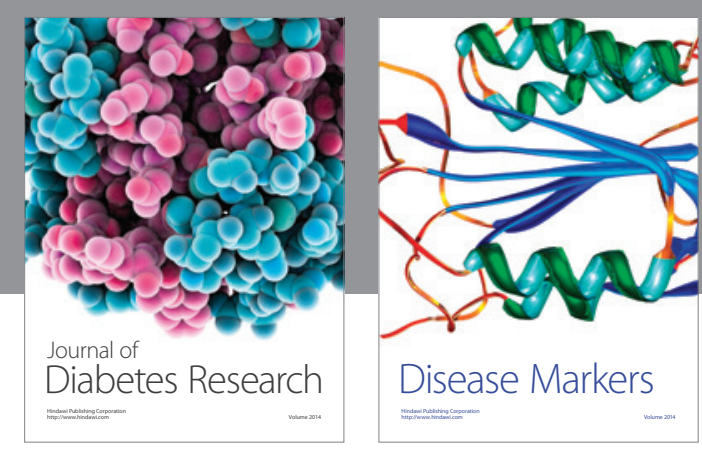

Disease Markers
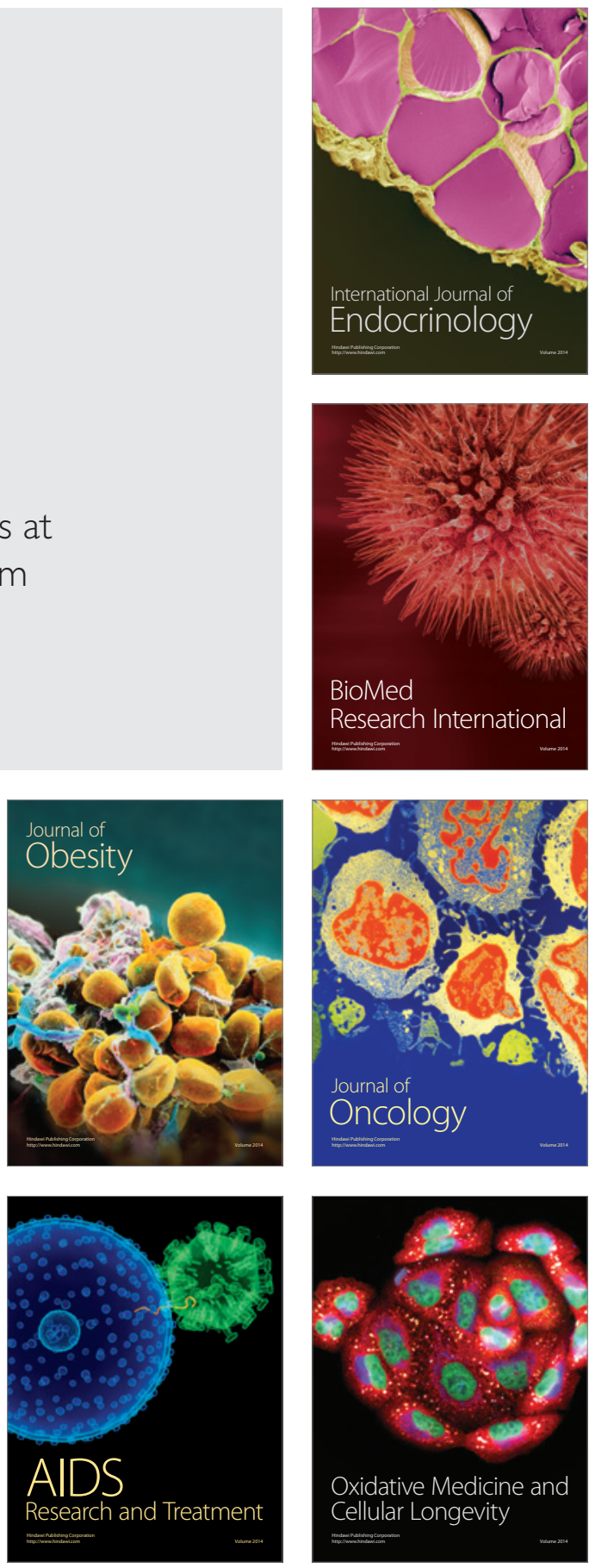